\title{
Estimating the uncertainty in passive-microwave rain-retrievals
}

\author{
Dorothée COPPENS*, Ziad HADDAD** and Eastwood IM** \\ *California Institute of Technology, Pasadena, CA 91106 , USA. \\ **Jet Propulsion Laboratory, Pasadena, CA 91106, USA. \\ email: dorothee@ vegemite.jpl.nasa.gov
}

\section{INTRODUCTION}

Current passive-microwave rain-retrieval methods are largely based on databases built off-line using cloud models. The vertical distribution of hydrometeors within the cloud has a large impact on upwelling brightness temperatures ([3],[5]). Thus, a forward radiative transfer model can predict off-line the radiance associated with different rain scenarios. To estimate the rain from measured brightness temperatures, one simply looks for the rain scenario whose associated radiances are closest to the measurements. To understand the uncertainties in this process, we first study the dependence of the simulated brightness temperatures on different hydrometeor size distribution (DSD) models. We then analyze the marginal and joint distributions of the radiances observed by the Tropical Rainfall Measuring Mission satellite and of those in the databases used in the TRMM rain retrievals. We finally calculate the covariances of the rain profiles and brightness temperatures in the TRMM passive-microwave database and derive a simple parametric model for the conditional uncertainty, given measured radiances. These results are used to characterize the uncertainty inherent in the passive-microwave retrieval.

\section{EFFECTS OF THE DSD ON THE RADIANCES}

Most radiative transfer models currently used to calculate the expected brightness temperatures $(T b)$ associated with rain events assume that the rain drops are distributed according to the Marsall-Palmer drop size distribution (DSD) (e.g. [4]),

$$
N(D) d D=N_{0} e^{-\Lambda D} d D
$$

with $N_{0}=0.08 \mathrm{~cm}^{-4}$ and $\Lambda=41 R^{-0.21} \mathrm{~cm}^{-1}$, where $R$ is the rain rate. If we calculate the rainfall from this equation, the result $\left(R_{\text {post }}\right)$ is quite different fron the original rainfall $R$ used to determine $\Lambda$. We therefore modified $N_{0}$ to make $R_{\text {post }}=$ $R$. The study has been done with these two Marshall-Palmer distributions. A different $\Gamma$-distribution was proposed in [1] :

$$
N(D)=N_{0}\left(R, S^{\prime \prime}, D^{\prime \prime}\right) D^{\mu\left(R, S^{\prime \prime}, D^{\prime \prime}\right)} e^{-\Lambda\left(R, S^{\prime \prime}, D^{\prime \prime}\right) D},
$$

with 3 uncorreleted variables: the rain rate $R$, the normalized mass-weighted mean drop diameter $D^{\prime \prime}$ and relative deviation $S^{\prime \prime}:$

$$
\begin{aligned}
\mu & =\frac{1}{s^{\prime 2} D^{\prime 0.33} R^{0.074}}-4 \\
\Lambda & =\frac{1}{s^{\prime 2} D^{\prime \prime 1.33} R^{0.23}} \\
N_{0} & =55 \frac{\Lambda^{\mu+4}}{\Gamma(\mu+4)\left(1-(1+0.53 / \Lambda)^{-\mu-4}\right)} R .
\end{aligned}
$$

Based on the TOGA/COARE data, we found $D^{\prime \prime}=1.13 \pm 0.32$ and $S^{\prime \prime}=0.39 \pm 0.025$. To compare the effets of these distributions, we first determined $S^{\prime \prime}$ and $D^{\prime \prime}$ in (2) to fit the original (MP0) and the adjusted (MP) Marshall-Palmer distribution, by minimizing the channels $10.7 \mathrm{GHz}$ and $37.0 \mathrm{GHz}$. Table I and figure I confirm that the Marshall-Palmer distribution with the adjusted $N_{0}$ is very close to distribution (2).

\begin{tabular}{|c||c|c||c|c|}
\hline \multicolumn{1}{|c||}{} & \multicolumn{2}{c|}{$0.5<R<5 \mathrm{~mm} / \mathrm{hr}$} & \multicolumn{2}{c|}{$5<R<50 \mathrm{~mm} / \mathrm{hr}$} \\
& $10.7 \mathrm{GHz}$ & $37.0 \mathrm{GHz}$ & $10.7 \mathrm{GHz}$ & $37.0 \mathrm{GHz}$ \\
\hline \hline MP0 - D & 1.24 & 1.04 & 1.26 & 1.16 \\
\hline MP $-D^{\prime \prime}$ & 1.14 & 1.13 & 1.17 & 1.12 \\
\hline \hline
\end{tabular}

TABLE I

Parameters which minimize the calculated $10.7 \mathrm{GHz}$ and $37.0 \mathrm{GHz}$ radiances.

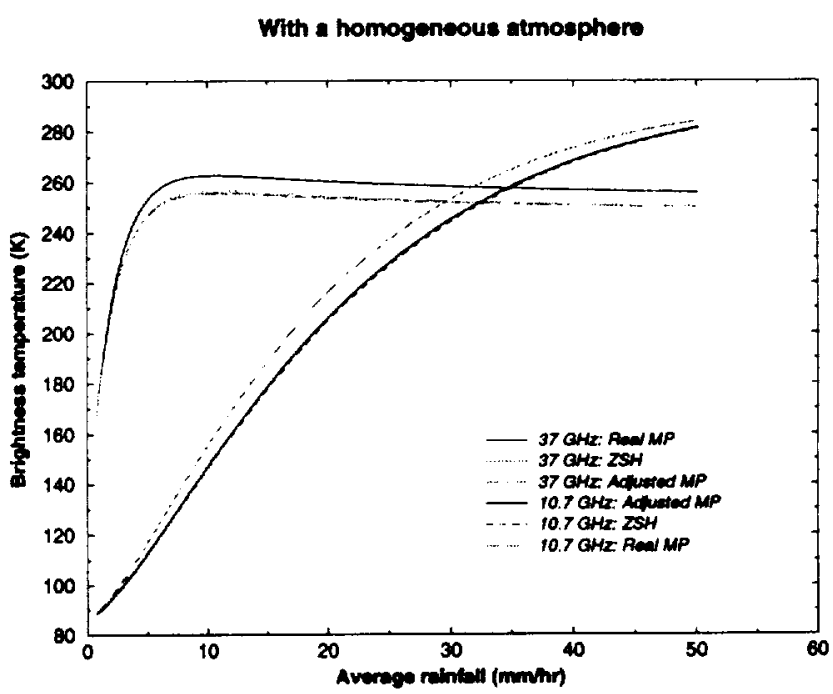

Fig. 1. Radiative transfer model calculation

Manifestly, the original and widely used Marshall-Palmer distribution implies a larger $D^{\prime \prime}$, hence larger drops for the same brightness temperatures. As figure 1 shows, the $10.7 \mathrm{GHz}$ based $R-T b$ correspondence under-estimates the rainfall by about $15 \%$ on average, whereas the $37 \mathrm{GHz}$-based correspondence under-estimates slightly at low rain rates but overestimates subtantially beyond about $8 \mathrm{~mm} / \mathrm{hr}$, when scattering effects become important. We found that the effect of the DSD was minimized near $16 \mathrm{GHz}$. These results are confirmed by cloud model simulations from the TRMM database, as figure 2 shows. 
$10.7 \mathrm{CHZ}$

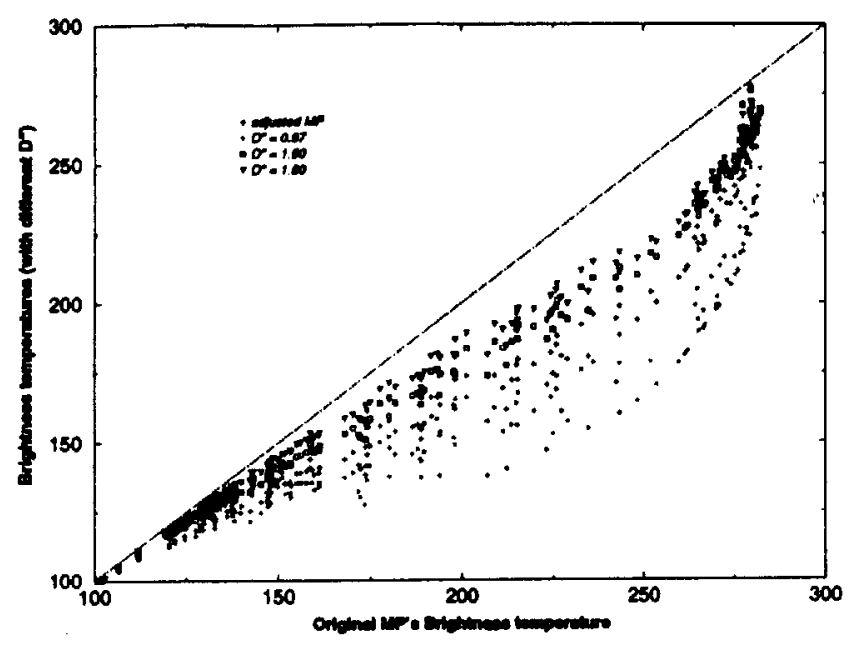

Fig. 2. Comparison between different DSD calculation.

\section{RE-PARAMETRIZATION OF $\boldsymbol{R}$}

Assume for simplicity that the typical atmosphere has 5 layers, with $R$ constant in each. To understand the joint behavior of the rain and the radiances, one must compute their covariance. If $R_{1}^{\prime}, \cdots, R_{5}^{\prime}$ are the 5 eigenvectors of the covariance matrix of $\log (R)$ calculated for the TRMM cloud simulations database,

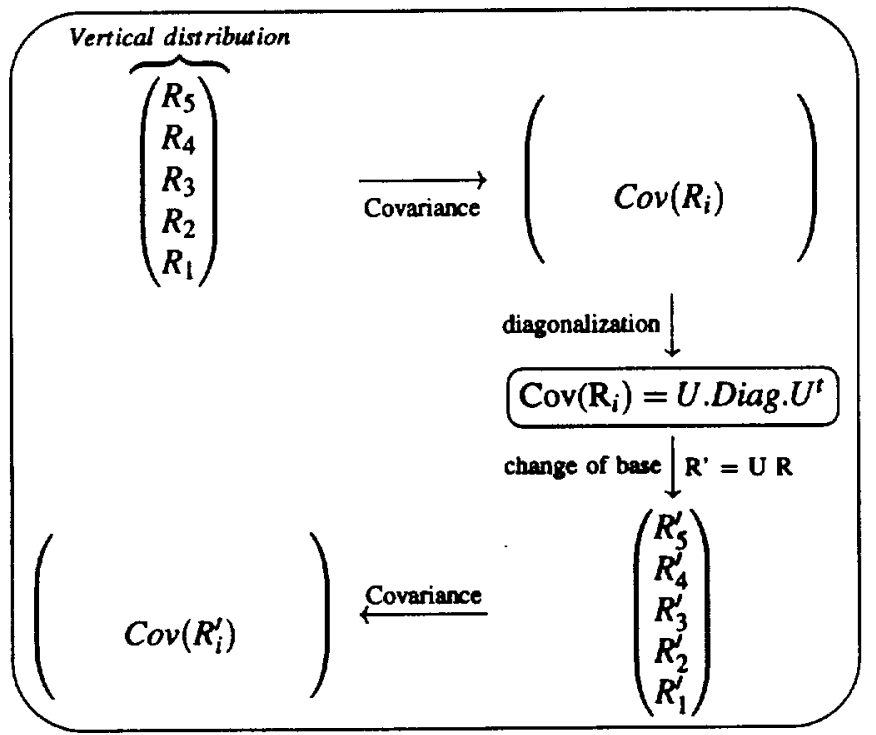

we found that $R_{1}^{\prime} \simeq \frac{1}{\sqrt{5}} \Sigma_{1}^{5} \log R_{i}$. Moreover, the eigenvalues are

$$
\operatorname{Cov}\left(R_{i}^{\prime}\right)=\left(\begin{array}{ccccc}
10 & 0 & . & . & 0 \\
0 & 1 & . & . & 0 \\
0 & . & 10^{-2} & . & 0 \\
0 & . & . & 10^{-2} & 0 \\
0 & . & . & 0 & 10^{-2}
\end{array}\right)
$$

This means that the entire rainfall distribution in the atmosphere can be described to first order by the vertically averaged rain rate $R_{1}^{\prime}$ and the constant values of the means of $R_{2}^{\prime}, \cdots, R_{5}^{\prime}$. When reconstructed in this way, the values for the rain rates were within $0.2 \%$ of the original values (see table II).

\begin{tabular}{|c||c|c|}
\hline & $\begin{array}{c}\text { rms deviation }(\mathrm{mm} / \mathrm{hr}) \\
\text { in } \mathrm{mm} / \mathrm{hr}\end{array}$ & relative deviation \\
\hline \hline$R_{1}$ & $8.9510^{-2}$ & \multirow{3}{*}{$1.2695310^{-3}$} \\
\cline { 1 - 2 }$R_{2}$ & $7.8910^{-2}$ & \\
\hline$R_{3}$ & $5.9810^{-2}$ & \\
\cline { 1 - 2 }$R_{4}$ & $4.0510^{-2}$ & \\
\hline$R_{5}$ & $2.6610^{-2}$ & \\
\hline
\end{tabular}

TABLE II

RMS error in the rainrate estimated for each layer from the mean rainrate $\left(R_{1}^{\prime}\right)$ and $E\left\{R_{2}^{\prime}\right\}, \cdots, E\left\{R_{5}^{\prime}\right\}$

This result, obtained from the TRMM cloud model database, is quite similar to the ones found using the TRMM radar data. Using five 1-km layers, the eigenvalues for convective events in October 1998 were $12.46>5.1>0.94>0.3>1.10^{-2}$ and the orthonormalized eigenvector $a_{i} \log R^{i}$ for the first eigenvalue 12.46 had $0.28<\left(a_{i}\right)<0.5$, which compares favorably with our predicted value $\frac{1}{\sqrt{5}} \simeq 0.447$. So the TRMM radar's $R_{1}^{\prime}$ is indeed also essentially the average rainfall. For stratiform events, the TRMM radar's eigenvalues were $7.6>$ $1.86>0.4>0.14>810^{-3}$, and the coefficients $a_{i}$ of $R_{1}^{\prime}$ were in the range $0.35<\left(a_{i}\right)<0.51$. In both cases, the eigenvector for the second value was $\left(a_{1}, a_{2}, a_{3},-a_{4},-a_{5}\right)$, with $0.22<a_{1}, a_{2}, a_{4}, a_{5}<0.52$ and $a_{3} \cong 0.05$. Thus, one can describe the rainfall to second order using the mean rain rate value and a simple difference between the rain in upper layers and that in lower layers.

\section{ESTIMATION OF $R$ FROM $T b$}

Since we can describe the vertical rainfall distribution to first order using a single variable $R_{1}^{\prime}$ and constants $\overline{R_{2}^{\prime}}, \cdots, \overline{R_{5}^{\prime}}$, the estimation problem is reduced to calculating the conditional mean and variance of $R_{1}^{\prime}$ from the brightness temperatures. To find the best relation between $R_{1}^{\prime}$ and a combination of the TRMM brightness temperatures $T^{i}$ at $10.7 \mathrm{GHz}, 19.3 \mathrm{GHz}$, $21.3 \mathrm{GHz}, 37 \mathrm{GHz}$ and $85.5 \mathrm{GHz}$, we maximized the expectation $E\left\{R_{1}^{\prime} . T^{\prime}\right\}$ keeping $E\left\{T^{\prime 2}\right\}$ constant, where:

$$
T^{\prime}=v_{i}\left(T^{i}-E\left\{T^{i}\right\}\right)
$$

The optimal $v_{i}$ minimize the scatter between $T^{\prime}$ and $R^{\prime}$. Once the $v_{i}$ are found, one can easily compute the mean and variance of $R^{\prime}$ given $T^{\prime}$. We considered many combinations of passive channels, from 5 to 2 brightness temperatures. The results were worst when we did not use the $10.7 \mathrm{GHz}$ channel or when we used two polarizations of the same frequency. We obtained reasonably good results using the five vertically polarized channels, namely an average R.M.S. uncertainty of $27.0 \%$ on the 


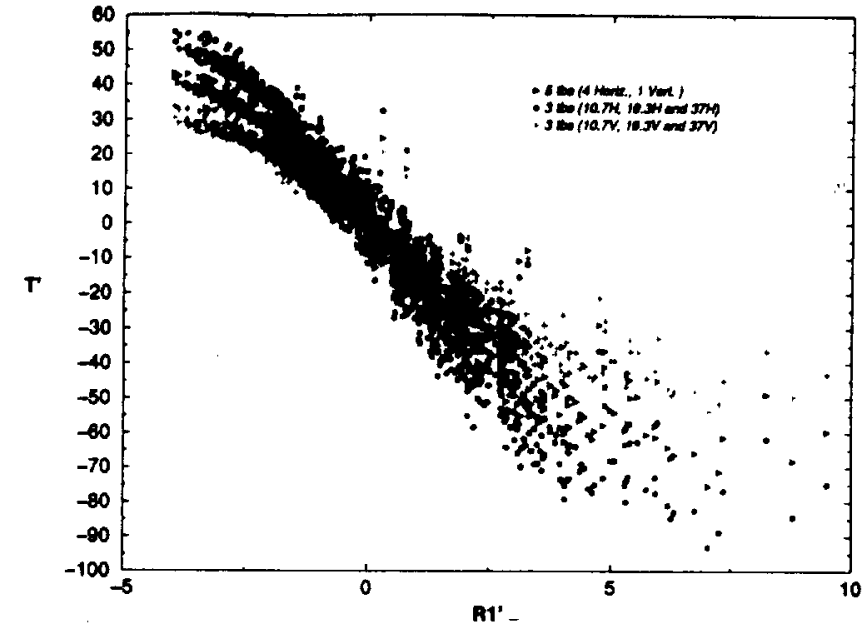

Fig. 3. Sample joint distribution, from the TRMM cloud model database, of $T^{\prime}$ and the log-average rain rate $R_{1}^{\prime}$.

mean rain rate. Our best results were obtained with horizontal polarizations, namely with

$$
\begin{aligned}
T^{\prime}= & 0.41 T_{10.7 H}+0.36 T_{19.3 H}+0.79 T_{21.3 \mathrm{~V}} \\
& -0.18 T_{37 H}-0.182 T_{85.5 H}
\end{aligned}
$$

for which the average R.M.S. uncertainty was $26.4 \%$ and with

$$
T^{\prime}=0.53 T_{10.7 H}+0.82 T_{19.3 H}-0.2 T_{37 H}
$$

for which the average R.M.S. uncertainty was $26.8 \%$.

Figures 3 and 4 illustrate the results obtained with the $T^{\prime}$ above. Figure 3 shows plots of $T^{\prime}$ versus $R_{1}^{\prime}$, for $T^{\prime}$ as in (7) and (8) above and for a sub-optimal $T^{\prime}$. Figure 4 shows the reconstructed near-surface rain rate $R_{1}$ plotted against the original.

\section{CONCLUSION}

Our study of the joint behavior of the rain in a horizontally stratified atmosphere and the associated microwave radiances shows that the single most crucial variable characterizing the rain profile is the vertically averaged rain rate, followed as a distant second by the difference between the high-altitude sub-freezing-level rain and the precipitation closer to the surface, the remaining eigen-variables having negligibly small variances implying that they can safely be considered constant (equal to their respective means). The study also shows that a judiciously chosen linear combination of the brightness temperatures can estimate the rain quite adequately, with an average R.M.S. uncertainty (due to the variations accounted for in the TRMM cloud model database) of about $27 \%$. The DSD does affect the brightness temperatures, and hence the eventual retrievals. Below $16 \mathrm{GHz}$, where scattering is not significant, the lager the mean drop size the smaller the rain associated with

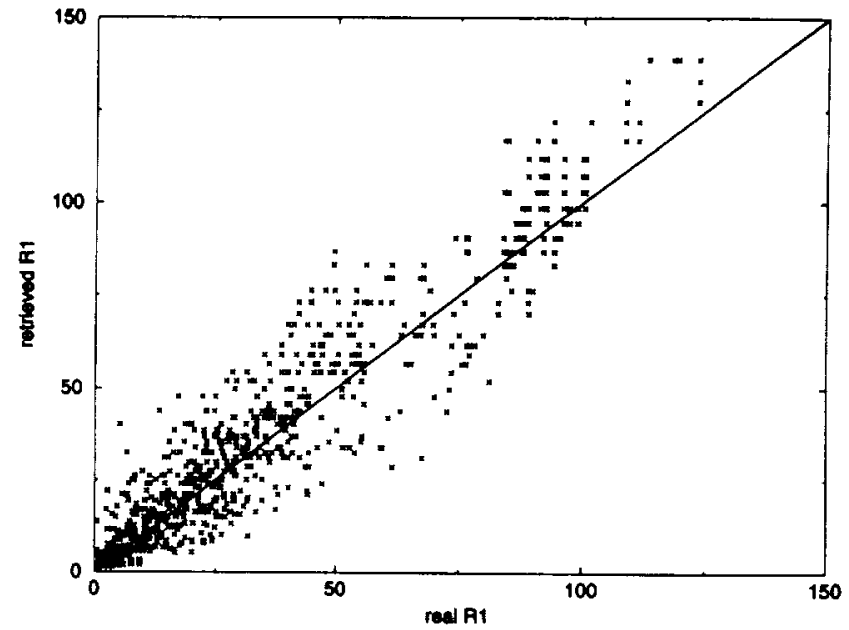

Fig. 4. $R_{1}$ (found from $T^{\prime}$ ) versus the original $R_{1}$, using 3 horizontal brightness temperatures $\left(T_{10.7}, T_{19.3}\right.$ and $\left.T_{37}\right)$.

a given brightness temperature. The effect is reversed above 16 $\mathrm{GHz}$ for higher rain rates.

\section{ACKNOWLEDGMENTS}

This work was performed at the Jet Propulsion Laboratory, California Institute of Technology, under contract with the National Aeronautics and Space Administration.

\section{REFERENCES}

[1] Z.S. Haddad, D. A. Short, S.L. Durden, E. Im, S. Hensley, M. B. Grable and R. A. Black, A new parametrization of the rain drop size distribution, IEEE Tran. geosc. remote sensing, vol. 35. no. 3, pp 532-539, May, 1997.

[2] Christian Kummerow, William S. Olson, Louis Giglio, A Simplified Scheme for Obtaining Precipitation and Vertical Hydrometeor Profiles from Passive Microwave Sensors, IEEE Tran. geosc. remote sensing, vol. 34. no. 5, pp 12131232, September, 1996.

[3] A. Mugnai, E.A. Smith and G.J. Tripoli, Foundations for statistical-physical precipitation retrieval from passive microwave frequencies. Part II: Emission-source and generalized weighting-function properties of a time-dependent cloud-radiation model, J. Appl. Meteo., vol. 32, pp 17-39, 1993.

[4] J. S. Marshall and W. M. Palmer, Relation of raindrop size to intensity, J. Meteor., vol. 5, pp 165-166, 1948.

[5] E.A. Smith, A. Mugnai, H.J. Cooper, G.J. Tripoli, X. Xiang, Foundations for statistical-physical precipitation retrieval from passive microwave frequencies. Part I: Brightness-temperature properties of a time-dependent cloud-radiation model, J. Appl. Meteo., vol. 31, pp 506531, 1992. 